\title{
Crescimento inicial de espécies arbóreas nativas em solos degradados e com presença de plintita no Bioma Cerrado, Brasília - DF
}

\author{
Edberto Moura LIMA ${ }^{1 *}$, Gustavo Ribas CURCIO ${ }^{1}$, Annete BONNET ${ }^{2}$, \\ Alexandre UHLMANN ${ }^{2}$, Viviane Helena PALMA ${ }^{1}$ \\ ${ }^{1}$ Programa de Pós-Graduação em Engenharia Florestal, Universidade Federal do Paraná, Curitiba, PR, Brasil. \\ ${ }^{2}$ Embrapa Florestas, Colombo, PR, Brasil \\ *E-mail: edberto.moura@gmail.com
}

Recebido em fevereiro/2018; Aceito em maio/2018.

\begin{abstract}
RESUMO: A intensificação do processo de antropização somado às características dos solos do Cerrado, em grande parte oligotróficos e intemperizados, dificulta a regeneração natural das espécies florestais, sendo necessária a intervenção humana para recompor a paisagem natural. O presente estudo teve como objetivo indicar espécies para iniciar o processo de recuperação de áreas degradadas do Cerrado com presença de plintita e processo de erosão estabelecido. Desta forma, analisamos as taxas de incremento em diâmetro e altura; e a taxa de sobrevivência e mortalidade de 963 indivíduos plantados próximo a área ciliar, em diferentes espaçamentos e com distinta composição de espécies. Os valores de incremento e sobrevivência variaram bastante de espécie para espécie. Das 17 espécies arbóreas plantadas oito apresentaram taxas de sobrevivência igual ou superior a $80 \%$. As taxas de incremento em altura e diâmetro foram classificadas em três grupos: baixo, médio e alto incremento. Quanto à altura, cerca de $50 \%$ das espécies testadas apresentaram de alto a médio incremento. Oito espécies tiveram valores de incrementos considerados baixos e duas espécies não foram mensuradas devido à elevada mortalidade. Para a distribuição dos incrementos diamétricos, com exceção de I. laurina todas as espécies apresentaram baixo valor. Em geral, os baixos valores de incrementos observados neste experimento são explicados, em grande parte, pelos fatores abióticos, principalmente pelas características edáficas da área. A ausência de horizonte A e o caráter petroplíntico reduzem o volume total de solo disponível a ser explorado pelas raízes, comprometendo o crescimento das plantas. Contudo, mesmo em condições ambientais desfavoráveis as espécies estudadas demostraram rusticidade. Entre as espécies arbóreas estudadas, T. roseoalba, M. urundeuva, A. aculeata, A. niopoides, G. ulmifolia, E. nitens, G. americana e I. laurina apresentaram taxa de sobrevivência de alta a média e com elevados incrementos. Essas características indicam que as espécies citadas são aptas a iniciar o processo de recuperação.
\end{abstract}

Palavras-chave: Regeneração, fragilidade, sobrevivência, Cerrado.

\section{Initial growth of native tree species in a degraded soil with presence of plinthite in Biome Cerrado, Brasília-DF}

\begin{abstract}
The intensification of the anthropization process, associate with the characteristics of the Cerrado soils, which are largely oligotrophic and weathered, are an impediment to natural regeneration of forest species, requiring human intervention to restore the natural landscape. The present study had as objectives to indicate species to initiate the process of recovery of degraded areas of the Cerrado with presence of plintite and established erosion process. In this way, we analyze the rates of increase in diameter and height; and the survival and mortality rate of 963 individuals planted near the ciliary area, at different spacings and with different species composition model. Increment and survival values varied greatly from species to species. Of the 17 trees species planted eight presented survival rates equal to or greater than $80 \%$. The rates of increase in height and diameter were classified into three groups: low, medium and high increment. Regarding the height, about $50 \%$ of the species tested showed high to medium increment. Eight species had values of increments considered low and two species were not measured due to the high mortality. For the distribution of the diametric increments, except for I. laurina all species showed low increment value. In general, the low values of increments observed in this experiment are explained, greatly, by the abiotic factors, mainly the edaphic characteristics of the area. The absence of horizon A and the petroplinthic character reduces the total volume of soil available to be explored by the roots, put at risk the plants growth rates. However, even under unfavorable environmental conditions, the studied species showed a rusticity. Among the arboreal species studied, $\mathrm{T}$. urumeuva, A. aculeata, A. niopoides, G. ulmifolia, E. nitens, G. americana and I. laurina presented a high to medium survival rate with high increases. These characteristics indicate that the species mentioned are apt to initiate the recovery process.
\end{abstract}

Keywords: Regeneration, fragility, plant species survival, Cerrado. 


\section{INTRODUÇÃO}

Ao longo dos séculos, a atividade humana vem provocando alterações na paisagem e degradação dos ecossistemas. Esses processos foram intensificados nas últimas décadas, devido ao aumento da capacidade tecnológica e avanço humano sobre áreas naturais, provocando a redução da cobertura vegetal, ameaçando a vida silvestre e comprometendo os serviços ecossistêmicos (NEWBOLD et al., 2016)

Segundo relatório da FAO; ITPS (2015) cerca de 33\% dos solos agricultáveis do mundo apresentam algum estágio de degradação, reduzindo significativamente a produtividade das culturas e a capacidade de armazenar carbono, nutrientes e água. A intensidade do processo de exploração e a frequência dos distúrbios comprometem a regeneração natural e a recuperação de ambientes degradados, até mesmo daqueles considerados altamente resilientes, como o Cerrado (DURIGAN, 2005).

O Cerrado, segundo maior bioma brasileiro, é formado por diferentes fisionomias distribuídas entre formações savânicas, florestais e campestres (WALTER et al., 2008). O bioma é considerado um hotspot global (MYERS et al., 2000) e está entre as áreas prioritárias para conservação nacional. Porém, apesar dos esforços para conservação o Cerrado está entre os biomas que mais sofreram alterações antrópicas, no Brasil, nas últimas décadas. Estudos do Ministério do Meio Ambiente revelaram que 54,5\% do bioma ainda mantém sua vegetação natural. Entretanto, esse percentual é preocupante já que somente $8,21 \%$ de sua área total está protegida sob a forma de unidades de conservação (BRASIL, 2015).

Devido à importância do Cerrado e sua pequena área protegida integralmente, existe grande preocupação quanto ao célere processo de ocupação desse ambiente e o uso descontrolado e inadequado dos recursos naturais. A ocupação e o uso do Cerrado, geralmente, estão associados à retirada da cobertura vegetal, interferindo nos diferentes ciclos biogeoquímicos e no equilíbrio dinâmico dos ecossistemas. Dentre os diversos processos afetados pela retirada da vegetação estão os mecanismos de transporte de água. A cobertura vegetal reduz a erosão e aumenta o potencial de infiltração, sendo fundamental para recarga dos aquíferos (REATTO et al., 2001).

O Cerrado é também conhecido por seus solos, em grande parte oligotróficos e intemperizados. As características edáficas do Cerrado são fundamentais para explicar a distribuição da vegetação na paisagem (MARTINS et al., 2015; MEWS, 2014; SOLLINS, 1998; SWANSON et al., 1988). Sendo assim, é importante observar, além da distribuição natural das espécies, quais seriam mais indicadas para plantio de acordo com o solo, pois, a escolha correta é um dos pontos mais críticos para a obtenção de sucesso durante o processo inicial de sucessão em área degradada (NERI et al., 2011).

Segundo Durigan et al. (2011), o plantio em áreas degradadas do Cerrado é indicado quando não se observa na área condições que favoreçam a regeneração natural, como a presença de caules subterrâneos e boas condições edáficas. A regeneração natural torna-se ainda mais desafiadora em áreas totalmente desmatadas e cultivadas intensamente por anos a fio. A partir desta observação surgem ao menos três questionamentos importantes, previamente elencados por Pereira et. al. (2015), necessários para embasar as metodologias de restauração, são eles: a) quais espécies plantar? b) quanto plantar de cada espécie e c) como efetivar este plantio, recobrindo o solo em menor tempo, com menores perdas e custo?

Este trabalho busca contribuir com respostas referentes à questão "a", buscando identificar e indicar espécies aptas a iniciar processo de recuperação de área degradada do Cerrado com presença de plintita e processo de erosão estabelecido.

\section{MATERIAL E MÉTODOS}

Esta pesquisa foi realizada em área de $4.752 \mathrm{~m}^{2}$ na Fazenda Entre Rios/DF (latitude 15 $57^{\prime} 33^{\prime}$ 'S, longitude 47²7’35,39',O), situada na área do Programa de Assentamento Dirigido do Distrito Federal (PAD/DF).

De acordo com classificação climática de Köppen, a área está sob influência do clima tropical com inverno seco (Aw). No período entre maio e setembro há baixa taxa de precipitação, apenas $1,5 \%$ do total anual, que é em média $1.500 \mathrm{~mm}$ (CARDOSO et al., 2014). Contudo, entre os anos 2012 e 2016 a média anual pluviométrica aferida na fazenda onde está alocado o experimento foi de $1.060 \mathrm{~mm}$, aproximadamente $30 \%$ inferior ao citado por Cardoso et al. (2014). Conforme esses autores, a temperatura ao longo do ano apresenta amplitude de $4,5{ }^{\circ} \mathrm{C}$, sendo que junho e julho têm média de temperatura de $20,8{ }^{\circ} \mathrm{C}$ e outubro, média de $25,3^{\circ} \mathrm{C}$.

Tabela 1. Propriedades físico-químicas do CambissoloHáplico Distrófico Petroplíntico (CXbd) e Neossolo Regolítico Distrófico petroplíntico (RRd), presentes na área experimental PAD/DF, Brasil.

Table 1. Physical-chemicalpropertiesoftheCambissoloHáplico Distrófico Petroplíntico (CXbd) andNeossolo Regolítico Distrófico petroplíntico (RRd), present in the experimental area PAD / DF, Brazil.

\begin{tabular}{|c|c|c|c|c|c|c|c|c|}
\hline \multirow{2}{*}{ Tipo } & \multirow{2}{*}{$\begin{array}{l}\text { Horizonte/ } \\
\text { Espessura }\end{array}$} & Areia grossa & Areia fina & Silte & Argila & $\mathrm{S}$ & CTC & \multirow{2}{*}{$\frac{\mathrm{V}}{\%}$} \\
\hline & & \multicolumn{4}{|c|}{$\mathrm{g} / \mathrm{kg}$} & \multicolumn{2}{|c|}{ cmolc. $\mathrm{dm}^{3}$} & \\
\hline \multirow{4}{*}{$\mathrm{CXbd}$} & Ap $0-26 \mathrm{~cm}$ & 85 & 96 & 324 & 495 & 1,33 & 5,93 & 22 \\
\hline & Bi $26-41 \mathrm{~cm}$ & 62 & 115 & 325 & 498 & 0,35 & 4,35 & 8 \\
\hline & $\mathrm{Bc} 41-78 \mathrm{~cm}$ & 51 & 116 & 343 & 490 & 0,33 & 9,33 & 4 \\
\hline & $\mathrm{Cr} 78-105 \mathrm{~cm}$ & 69 & 98 & 383 & 450 & 0,23 & 9,23 & 2 \\
\hline \multirow{2}{*}{$\mathrm{RRd}$} & Ap $0-20 \mathrm{~cm}$ & 294 & 163 & 143 & 400 & 1,43 & 6,83 & 21 \\
\hline & C $20-36 \mathrm{~cm}$ & 54 & 40 & 431 & 475 & 0,45 & 6,25 & 7 \\
\hline
\end{tabular}

Legenda: g: grama; kg: quilograma; S: soma de bases trocáveis; CTC: capacidade de troca catiônica; V: saturação por bases, cmolc: centimol de carga; dm: decímetro. 
$\mathrm{Na}$ área de plantio predomina a associação de Cambissolo Háplico Distrófico petroplíntico (CXbd) e Neossolo Regolítico Distrófico petroplíntico (RRd), ambos de textura argilosa. Embora sejam solos taxonomicamente distintos, são funcionalmente similares, em termos de resistência ao desenvolvimento das plantas e não apresentaram fonte de variação nas análises. O relevo local é predominantemente forte ondulado, com declividade variando entre $20 \%$ a $45 \%$.

O pré-plantio compreendeu na marcação dos locais das covas com estacas de madeira. Posteriormente, realizou-se coroamento, coveamento e roçada nas entrelinhas (Figura 1). $\mathrm{O}$ controle de formigas com iscas formicidas à base de sulfluramida foi realizado de maneira pontual, ou seja diretamente em cada foco, na dosagem de $8 \mathrm{~g} /$ metro $(\mathrm{m})^{2}$ de terra solta para formigas do gênero Atta (saúvas) ou 8 g/formigueiro do gênero Acromyrmex (quenquéns).

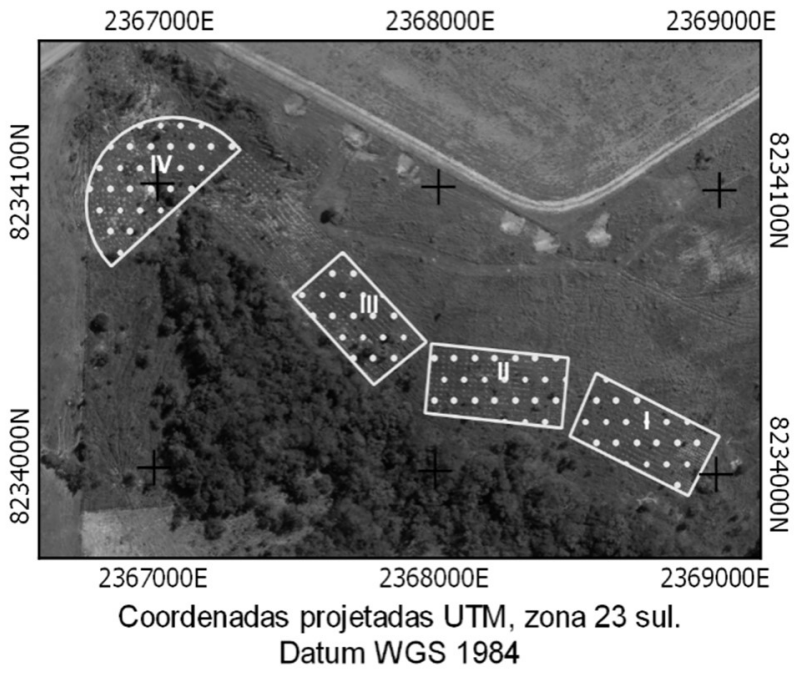

Figura 1. Disposição dos blocos experimentais, I a IV, ao longo da faixa ciliar, na propriedade Entre Rios PAD/DF, Brasil. Destaque para vários pontos com solo com evidentes sinais de erosão. Figure 1. Arrangement of the experimental blocks, I to IV, along the riparian area, at Fazenda Entre Rios PAD / DF, Brazil.

As covas foram abertas com motocoveadora nas dimensões de 40 centímetros $(\mathrm{cm})$ de profundidade por 20 $\mathrm{cm}$ de diâmetro. A adubação de base, por cova, consistiu de $80 \mathrm{~g}$ de termofosfato, $10 \mathrm{~g}$ de micronutrientes e $20 \mathrm{~g}$ de uréia Em cada cova foi adicionado 1 litro (1) de hidrogel hidratado na proporção $4 \mathrm{~g}$ de pó/1 1 de água.

O plantio do experimento foi realizado em novembro de 2012, ao longo de uma faixa dividida em três blocos de $50 \mathrm{~m}$ x 24 m (blocos I, II e III) com 576 mudas cada, e por outra área com plantio de 387 mudas realizado radialmente em razão da conformação e declive da área (bloco IV). O replantio das mudas foi realizado um mês após o plantio.

A seleção das espécies baseou-se na ocorrência natural na região e nas características ecológicas (Tabela 2). Estas foram agrupadas por módulos de plantio arranjados no espaço de acordo com espaçamento do sub-bloco. O bloco I continham módulos compostos por oito espécies, o bloco II por quatro espécies, o bloco III por uma espécie e o bloco IV por onze espécies dispostas aleatoriamente (Tabela 2). Os três primeiros blocos foram subdivididos em diferentes espaçamentos $(2 \times 2 \mathrm{~m}, 2 \times 1 \mathrm{~m}$ e $1 \times 1 \mathrm{~m})$ enquanto no quarto bloco, com plantio radial ao longo de 15 linhas, as mudas foram espaçadas por $2 \mathrm{~m}$ entre si.

Os tratamentos silviculturais de manutenção consistiram em dois coroamentos manuais e duas roçadas semimecanizadas por ano durante os dois primeiros anos. Nessas atividades também se realizava proteção das coroas das mudas com cobertura morta. No terceiro ano se realizou apenas uma roçada semimecanizada. O monitoramento e combate às formigas foram realizados durante três anos por meio de ronda mensal e, quando constatada a necessidade de combate, procedia-se com a dosagem descrita acima, de maneira pontual.

\subsection{Coleta e análise dos dados}

Entre os anos de 2013 a 2016 foram coletados dados de sobrevivência, diâmetro a altura do solo (DAS) e altura total (HT). O DAS e HT foram coletados com auxílio do paquímetro digital e vara graduada, respectivamente. A taxa de sobrevivência das espécies foi calculada conforme a equação 1:

$$
\operatorname{TS}(\%)=((\mathrm{N}-\mathrm{Nm}) / \mathrm{N}) \times 100
$$

(Equação 1)

em que: TS $(\%)=$ taxa de sobrevivência percentual; $\mathrm{N}=$ número de mudas plantadas; $\mathrm{Nm}=$ número de mudas mortas durante o período de avaliação.

Os incrementos diamétricos e em altura foram avaliados com base no crescimento das plantas medidos no intervalo entre 2014 e 2016, levando em consideração somente os indivíduos vivos no último período.

Durante as análises descartamos a influência dos espaçamentos sobre o crescimento em altura e diâmetro, uma vez que o período foi insuficiente para proporcionar competição nutricional e sombreamento. Desta forma, após serem verificadas as premissas estatísticas optou-se pelo uso da estática descritiva, para descrever o comportamento das variáveis de interesse.

\section{RESULTADOS}

As taxas de sobrevivência variaram bastante entre espécies e indivíduos, influenciando a média geral da espécie. Assim observamos a amplitude de 0 a $98 \%$ nos dados dessa variável. Contudo, a taxa de sobrevivência média total do experimento manteve-se entorno de $60 \%$ (Figura 2 ).

Seguindo a classificação proposta por Duboc; Guerrini, (2007), observamos oito espécies com alta taxa de sobrevivência, ou seja, um pouco mais de $50 \%$ das testadas apresentaram taxa de sobrevivência igual ou superior a $80 \%$. Duas espécies apresentaram taxas de sobrevivência média e sete foram classificadas com baixa taxa de sobrevivência (Figura 2). Dessas sete espécies cinco apresentaram taxas críticas de mortalidade, com destaque para $J$. spinosa e $Z$. rhoifolium que tiveram $100 \%$ de mortalidade no período final. 
Tabela 2. Espécies arbóreas implantadas na área experimental, situada na Fazenda Entre Rios PAD/DF - Brasil, segundo suas famílias, grupo ecológico e módulo de plantio.

Table 2. Species planted in the experimental area, located at Fazenda Entre Rios PAD / DF - Brazil, according to their families, ecological group and planting module

\begin{tabular}{|c|c|c|c|c|c|c|}
\hline Espécie & Família & Grupo ecológico* & Bloco I & Bloco II & Bloco III & Bloco IV \\
\hline Acrocomia aculeata (Jacq.) Lodd. ex Mart. & Caricaceae & $\mathrm{P}^{1}$ & & & & $\mathrm{X}$ \\
\hline Albizia niopoides(Spruce ex Benth.) Burkart & Fabaceae & $\mathrm{P}_{\mathrm{a} \mathrm{ST}}{ }^{2}$ & & & & $\mathrm{X}$ \\
\hline Anadenanthera colubrina (Vell.) Brenan & Fabaceae & $\mathrm{P}^{3}$ & $\mathrm{X}$ & $\mathrm{X}$ & & \\
\hline Cariniana rubra Gardner exMiers & Lecythidaceae & $\mathrm{C}^{4}$ & & & & $\mathrm{X}$ \\
\hline Copaifera langsdorffii Desf. & Fabaceae & $\mathrm{ST}$ a $\mathrm{C}^{3}$ & $\mathrm{X}$ & $\mathrm{X}$ & & \\
\hline Cybistax antisyphilitica (Mart.) Mart. & Bignoniaceae & $\mathrm{P}^{1}$ & $\mathrm{X}$ & & & \\
\hline Emmotum nitens (Benth.) Miers & Icacinaceae & $\mathrm{SI}$ a ST ${ }^{4}$ & & & & $\mathrm{X}$ \\
\hline Genipa americana $\mathrm{L}$. & Rubiaceae & $\mathrm{P}_{\text {a ST}}^{3}$ & $\mathrm{X}$ & & & \\
\hline Guazuma ulmifoliaLam. & Malvaceae & $\mathrm{P}$ a $\mathrm{C}^{5}$ & & & & $\mathrm{X}$ \\
\hline Handroanthus impetiginosus (Mart. ex DC.) Mattos & Bignoniaceae & $\mathrm{ST}$ a $\mathrm{C}^{3}$ & $\mathrm{X}$ & $\mathrm{X}$ & & \\
\hline Inga laurina $(\mathrm{Sw}$.$) Willd.$ & Fabaceae & $\mathrm{P}^{4}$ & $\mathrm{X}$ & $\mathrm{X}$ & $\mathrm{X}$ & $\mathrm{X}$ \\
\hline Jacaratia spinosa (Aubl.) A.DC. & Caricaceae & $\mathrm{P}$ a ST & $\mathrm{X}$ & & & \\
\hline Myracrodruon urundeuvaAllemão & Anacardiaceae & $\mathrm{SI}^{3}$ & & & & $\mathrm{X}$ \\
\hline Myrsine gardneriana A.DC. & Primulaceae & $\mathrm{P}^{6}$ & & & & $\mathrm{X}$ \\
\hline Simarouba amara Aubl. & Simaroubaceae & $\mathrm{SI}$ a $\mathrm{C}^{3}$ & $\mathrm{X}$ & & & \\
\hline Tabebuia roseoalba (Ridl.) Sandwith & Bignoniaceae & $\mathrm{P}^{6}$ & & & & $\mathrm{X}$ \\
\hline Zanthoxylum rhoifoliumLam. & Rutaceae & $\mathrm{P}$ a $\mathrm{C}^{5}$ & & & & $\mathrm{X}$ \\
\hline
\end{tabular}

Legenda: *P=pioneira; SI=secundária inicial; ST=secundária tardia; C=clímax; ${ }^{2}$ LORENZI (1992); ${ }^{2}$ CARVALHO(2008); ${ }^{3}$ CARVALHO (2003); ${ }^{4}$ LORENZI (1998); ${ }^{5}$ CARVALHO (2006); ${ }^{6}$ SANTOS; SUGAHARA; TAKAKI (2005)

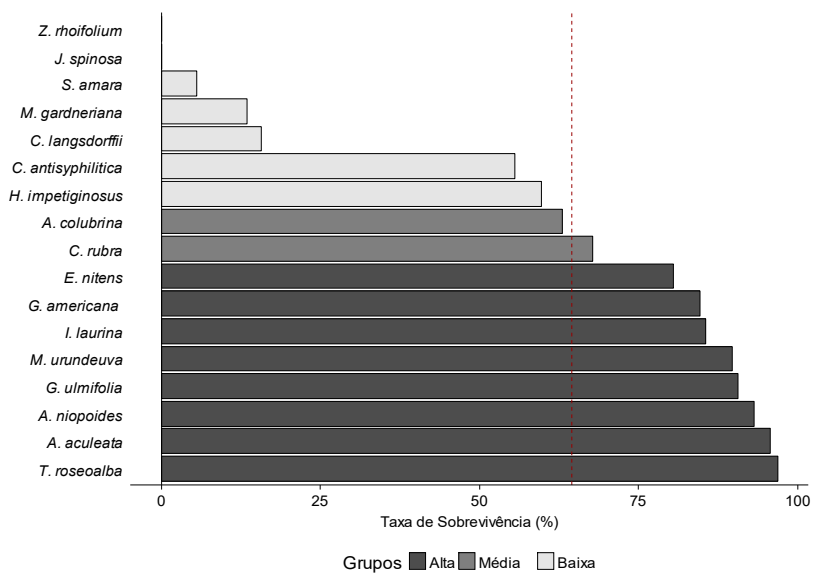

Figura 2.Taxa de sobrevivência média por espécie avaliada após 29 meses de instalação do experimento na Fazenda Entre Rios PAD/DF - Brasil. As espécies foram classificadas por grupos de sobrevivência de acordo com os critérios estabelecidos por Duboc; Guerrini (2007). A linha tracejada representa a taxa de sobrevivência média total do experimento.

Figure 2. Average survival rate per species evaluated after 29 months of installation of the experiment at the Fazenda Entre Rios PAD / DF - Brazil. The species were classified by survival groups according to the criteria established by Duboc; Guerrini (2007). The dashed line represents the overall mean survival rate of the experiment.

Apesar da elevada variação nas taxas de incremento, podemos dividir a altura em três grupos: espécies com alto valor de incremento (acima de $40 \mathrm{~cm}$ ), médio (entre 20 a 40 $\mathrm{cm})$ e baixo $(<20 \mathrm{~cm})$, conforme Figura 3 B. Dentro destes grupos, cerca de $50 \%$ das espécies testadas estão nos grupos de alto a médio e oito no grupo de baixo incremento, sendo que duas espécies não tiveram seu crescimento mensurado devido à elevada mortalidade.

Para a distribuição dos incrementos diamétricos, não observamos padrões de agrupamento nítidos como aqueles presentes nos incrementos de altura (Figura 3 A). Dentre todas as espécies analisadas somente I. laurina apresentou incrementos acima da média total, todas as demais apresentaram baixos valores de incremento diamétricos, algumas chegando a valores nulos (A. aculeata, S. amara, entre outras).

\section{DISCUSSÃO}

Uma das maneiras de avaliarmos o sucesso dos projetos de recuperação, principalmente na fase inicial, é por meio do monitoramento das taxas de mortalidade/sobrevivência dos indivíduos, sabendo que essas taxas são variáveis entre espécies e indivíduos (BRANCALION; RODRIGUES, 2015). Em estudo de recuperação em áreas do Cerrado no Distrito Federal, Cortes (2012) registrou taxas médias de sobrevivência entorno de $90 \%$ após 12 meses do plantio. Valores próximos (88\%) foram observados por Oliveira et al. (2015) em uma área de Cerrado de Brasília e por Almeida et al., (2016) (76,38\%) em uma área localizada em DiamantinaMG após período semelhante. Contudo, altas taxas de sobrevivência nem sempre são observadas em projetos de recuperação. Rezende (2004), ao analisar 88 propriedades localizadas no Distrito Federal, encontrou taxa média de sobrevivência próxima de $16,26 \%$.

Os resultados dessa pesquisa mostram que ao final de 29 meses a taxa média total de sobrevivência das espécies esteve próxima de $60 \%$ (Figura 1), logo, os valores encontrados nos trabalhos citados apresentam amplitude na qual nossos resultados estão compreendidos. Porém, mesmo apresentando valores incorporados aos da literatura, as taxas de sobrevivência estão abaixo dos valores recomendados por Almeida; Sánchez (2005) e Piña-Rodrigues et. al (1997), para plantios que visem à restauração de áreas - entre $80 \%$ a $90 \%$.

Outro ponto em destaque é a elevada amplitude nos valores de sobrevivência para cada espécie, as taxas variaram entre 0 a 98\% (Figura 1). Amplitudes semelhantes foram descritas por Almeida et al., (2016); Cortes, (2012); Silva; Corrêa (2008). Entretanto, esses valores não são consenso na literatura. Por exemplo, Martinotto et al. (2012) em pastagens degradadas no bioma Cerrado, após 20 meses de plantio, registraram amplitudes variando entre 79 a $100 \%$ nas taxas de sobrevivência. 

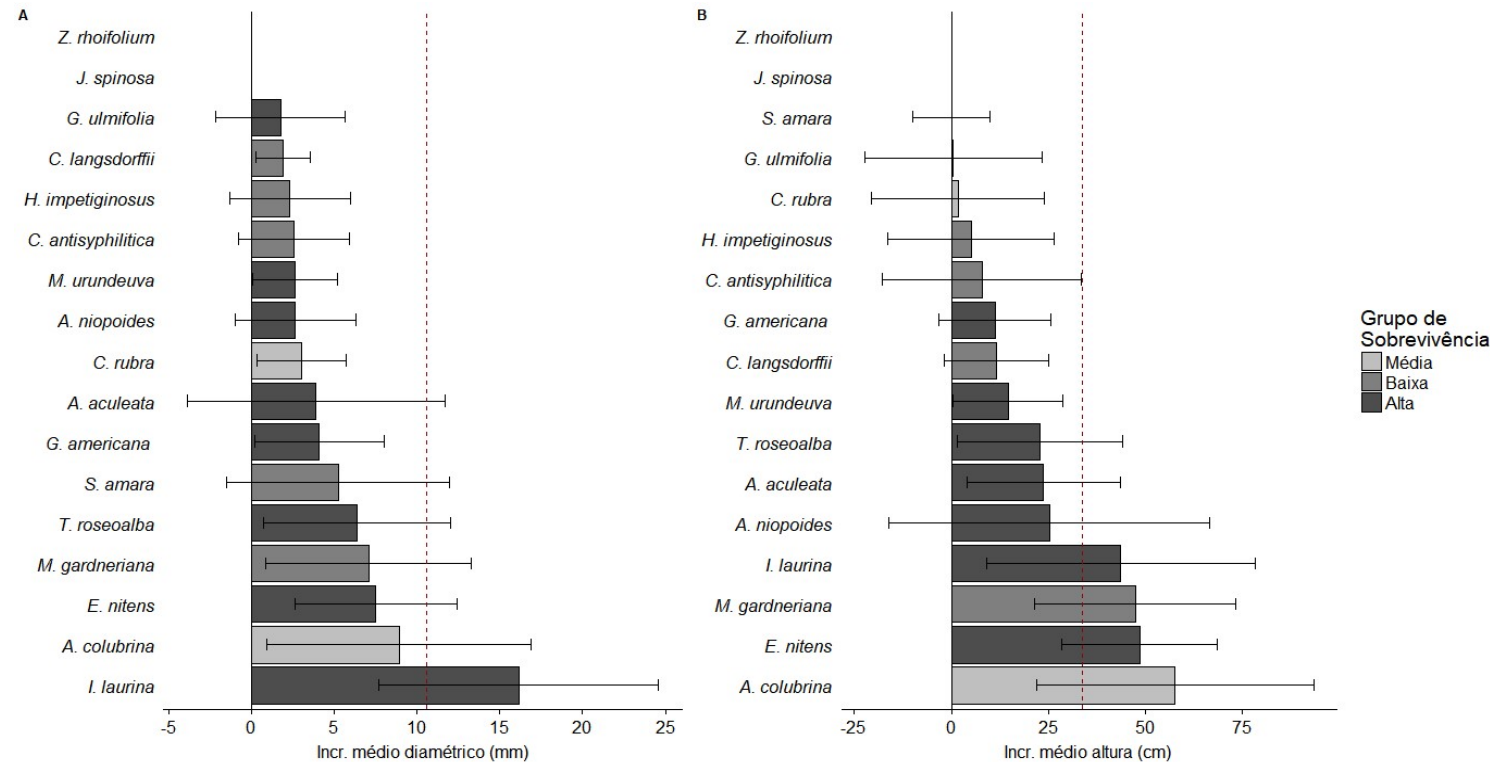

Figura 3. Incrementos mensurados após 29 meses de instalação do experimento na Fazenda Entre Rios PAD/DF - Brasil. A) incremento diamétrico $(\mathrm{mm})$ médio com desvio padrão por espécie; B) incremento altura $(\mathrm{cm})$ médio com desvio padrão por espécie. As espécies foram classificadas por grupos de sobrevivência de acordo com os critérios estabelecidos por Duboc; Guerrini (2007). E a linha tracejada representa os incrementos médios totais do plantio.

Figure 3. Increments measured after 29 months experiment installation at Fazenda Entre Rios PAD / DF - Brazil. A) mean diameter increment $(\mathrm{mm})$ with standard deviation by species; B) increment height $(\mathrm{cm})$ with standard deviation by species. The species were classified by survival groups according to the criteria established by Duboc; Guerrini (2007). The dashed line representes the total average planting increments.

Observamos uma relação entre as taxas de sobrevivência e as características dos grupos sucessionais (Tabela 2). A maioria das espécies enquadradas em alta sobrevivência possuem características de espécies pioneiras ou secundárias iniciais, altamente adaptadas a solos drenados e/ou pedregosos: T. roseoalba (SANTOS et al., 2005); $M$. urundeuva (GUSSON et al., 2011); A. aculeata (LORENZI, 1992); A. niopoides (CARVALHO, 2009); G. ulmifolia (POTT; POTT, 2002) e E. nitens (FELFILI et al., 2000). Algumas espécies desse grupo possuem características de plasticidade, como o caso da G. americana. Essa espécie é encontrada tanto em solos mal drenados amazônicos como em bem drenados, no Cerrado (PIRES, 2015). Outra espécie de elevada plasticidade é I. laurina, indicada para compor plantios de recuperação de áreas degradadas (BILIA et al. 2003).

No grupo de sobrevivência média, A. colubrina, é comumente relacionada como espécie de alta sobrevivência em plantios de recuperação de áreas no Cerrado (OLIVEIRA et al., 2015; ANTEZANA, 2008). Nessa pesquisa a referida espécie teve crescimento e sobrevivência aquéns aos trabalhos citados. Estes valores são justificados, em parte, pelas as características edáficas. O solo raso e erodido, além da presença expressiva de plintitas, podem ter contribuído para o baixo valor de incremento, não somente desta espécie, mas como de outras a exemplo da C. rubra e demais espécies do grupo com baixa sobrevivência.

Entre as espécies com menores taxas de sobrevivência, $H$. impetiginosus e C. antisyphilitica, apresentaram, respectivamente 55,6 e $60 \%$, frente a resultados inferiores a $6 \%$ para S. amara, Z. rhoifolium e J. spinosa. As duas primeiras mencionadas, são constantemente citadas em trabalhos de recuperação de áreas degradadas (FERREIRA et al., 2009; BARBOSA et al., 2013; DARONCO et al., 2013; ARAÚJO et al., 2014; MEIRA JUNIOR et al., 2015). No caso de $C$. langsdorffii é interessante salientar que em pesquisa de Silva Júnior et al. $(1998 ; 2001)$ é a segunda espécie mais frequente em ambientes de Matas de Galeria do Cerrado, que são mais úmidos que seus adjacentes, como é o caso do ambiente desta pesquisa. A M. gardneriana é descrita como espécie potencial para recuperação de áreas de exploração de minério de ferro (MEIRA JUNIOR et al., 2015), contudo neste estudo a espécie não apresentou crescimento semelhante àqueles descritos na literatura, mesmo em ambientes extremamente adversos como as áreas de mineração, que geralmente-são desprovidas de horizonte A.

Cabe destacar que espécies dos gêneros Tabebuia e Handroanthus, conhecidas como "ipês", além de $M$. urundeuva (aroeira), estão entre as madeiras de valor mais citadas para uso em pequenas propriedades rurais na região do Cerrado de Goiás (BONNET; CURCIO, 2016). Portanto, sendo espécies importantes a se considerar em projetos de plantios para utilização da madeira em áreas de recomposição de reserva legal, plantios de produção ou ainda de recomposição de áreas de preservação permanentes (APPs), neste último caso, permitido apenas em pequenas propriedades (BRASIL, 2012).

Quanto ao crescimento diamétrico, a maioria das espécies apresentou um crescimento líquido menor que $5 \mathrm{~mm}^{\mathrm{ano}} \mathrm{o}^{-1}$. Os valores observados estão abaixo daqueles descritos em outros estudos. Por exemplo, em um plantio realizado ao redor de um reservatório no Município de Indianópolis, MG, Lima et. al. (2009) relataram que após 13 meses as espécies tiveram um incremento médio entre 0,9 e $40,9 \mathrm{~mm}$, sendo que cerca de $70 \%$ das espécies estudadas apresentaram um incremento 
inferior a $10 \mathrm{~mm}$. Valores semelhantes foram observados por Cortes (2012), após 12 meses de plantio, onde o incremento médio variou entre $0,98 \mathrm{~mm}$ a $23 \mathrm{~mm}$.

O mesmo ocorreu para os incrementos em altura. A média geral observada de aproximadamente $14 \mathrm{~cm} \mathrm{ano}^{-1}$ está longe daquelas descritas nos trabalhos de Cortes (2012) e Lima et al. (2009). Outro ponto a ser destacado foi a diferença entre os valores médios observados por espécie e o valor médio total. Quando comparados observamos que somente quatro espécies (A. colubrina, E. nitens, $M$. gardneriana e I. laurina), tiveram um incremento médio maior que a média total (Figura 2).

Estudos de Felfili et al. (2000), demonstraram que em condições naturais e de baixa competição, plantas jovens podem crescer em altura, entre $0,5 \mathrm{~m}$ a $1 \mathrm{~m}$ ano-1. Apesar destes valores, o crescimento das árvores varia fortemente entre indivíduos, espécies, sítio e no tempo, sendo o crescimento uma resposta a ações simultâneas de uma infinidade de ciclos distintos de fatores abióticos e bióticos (BATISTA, 2014).

Em áreas degradadas, o crescimento e estabelecimento de espécies depende de diversos fatores como competição por água, nutrientes e luminosidade, bem como a predação por animais, entretanto, as condições edáficas do ambiente degradado provavelmente estão entre os fatores de maior impacto no estabelecimento das plantas (CARNEVALI et al. 2016). Neste estudo, as diferenças observadas nos resultados dos incrementos ocorreram em função das interações com os fatores abióticos, principalmente fatores edáficos. Os solos da área apresentam fase erodida, ou seja, ausência de horizonte A, e caráter petroplíntico o que reduz o volume total de solo disponível a ser explorado pelas raízes, tanto para desenvolvimento quanto para exploração de bases. Além disso, esses solos possuem baixos valores $\mathrm{V} \%$ e caráter oligotrófico, sobretudo devido à baixa CTC (Tabela 1).

Acreditamos que ao longo dos anos a associação de espécies da família Fabaceae com as demais espécies possa promover melhorias nas características físico-química dos solos da área de estudo. Segundo Claassen; Carey (2007) em solos degradados a liberação gradual do nitrogênio através da adição de material orgânico pode favorecer melhorias na qualidade físico-químico dos solos e auxiliar no estabelecimento e crescimento de outras plantas. Fabaceaes, em geral, são conhecidas devido à sua capacidade de fixar nitrogênio e, desta forma, apresentam maior concentração deste nutriente em seus tecidos do que plantas que não realizam este processo (VITOUSEK et al., 2010). Assim, a decomposição de seus tecidos leva ao aumento da disponibilidade deste elemento nas proximidades da planta.

Além disto, é importante ressaltar que, conforme Puig (2008), a área explorada pelo sistema radicular de uma árvore é considerável e frequentemente mais vasta que a projeção no solo da copa e, desta forma, a área total passível de ser beneficiada por essa interação é potencializada. Aspectos como esses são importantes principalmente em áreas muito degradas, onde a restauração do ambiente é demorada e trabalhosa, sendo necessárias diversas intervenções para garantir o sucesso do plantio.

Apesar de parte dos projetos de recuperação estarem preocupados com o rápido crescimento das plantas, a melhoria das condições edáficas a longo prazo, é fundamental para restabelecimento das principais funcionalidades ambientais e promover a colonização da área por espécies nativas.

Neste sentido, o uso de plantas de diversos grupos ecológicos e diferentes taxas de desenvolvimento aumentam as chances de sucesso dos plantios, principalmente em relação à restauração de funcionalidades ambientais. Desta forma, as espécies escolhidas para a região em questão, mesmo com os baixos incrementos, possivelmente relacionados às condições edafoclimáticas, tem demonstrado elevada rusticidade, ou seja, adaptadas às condições adversas. Além disso, algumas espécies têm demonstrado, precocemente, a capacidade de retomar as funcionalidades ambientais da área, tais como produção de frutos, atraindo a fauna local e cobertura dos solos, diminuindo competição com exóticas e aumentando deposição de matéria orgânica.

\section{CONCLUSÕES}

A variação nas taxas de sobrevivência e incrementos é comum em projetos de restauração. Contudo, valores baixos podem indicar problemas nos plantios dos projetos de restauração. Desta forma, os valores de incrementos observados neste experimento são explicados, em grande parte, por sua característica edáficas - solos pobres, do ponto de vista agronômico, e com presença de plintita reduzindo o volume de solo disponível para exploração do sistema radicular das plantas.

Mesmo em condições ambientais desfavoráveis as espécies estudadas demostraram rusticidade, característica fundamental em projetos de recuperação onde o ambiente está em estágio avançado de degradação. Além disso, observamos o restabelecimento de funcionalidades ambientais, por meio do desenvolvimento precoce de algumas espécies. Desta forma, as espécies listadas nos grupos de alta e média taxa de sobrevivência e com elevados incrementos, a exemplo $T$. roseoalba, M. urundeuva, $A$. aculeata, A. niopoides, G. ulmifolia, E. nitens, G. americana e I. laurina - são espécies a serem consideradas na implementação de plantios que visem a recuperação de áreas semelhantes ao do experimento.

\section{AGRADECIMENTOS}

Os autores gostariam de agradecer a Confederação Brasileira da Agricultura e Pecuária (CNA), a Empresa Brasileira de Pesquisa Agropecuária (Embrapa Florestas), o Projeto Biomas (Projeto Biomas) para a assistência financeira e apoio a esta pesquisa e a todos colaboradores e pesquisadores envolvidos. Agradecemos, também, o apoio da Coordenação de Aperfeiçoamento de Pessoal de Nível Superior - Brasil (CAPES) - Código de Financiamento 001.

\section{REFERÊNCIAS}

ALMEIDA, R. O. P. O.; SÁNCHEZ, L. E. Revegetação de áreas de mineração: critérios de monitoramento e avaliação do desempenho. Revista Árvore, Santa Maria, v. 29 , n. 1, p. 47-54, fev. 2005. DOI: http://dx.doi.org/10.5902/1980509815742

ALMEIDA, A. C. de; PEREIRA, I. M.; SILVA M. A. P. da; CARLOS, L.; SOUZA, F. M. de. Sobrevivência e crescimento de cinco espécies do cerrado em diferentes adubações na recuperação de uma cascalheira, Diamantina-MG. Enciclopédia Biosfera, Jandaia, v. 13, n. 23 , p. 1705-1716, jun. 2016. DOI: 
http://dx.doi.org/10.18677/Enciclopedia_Biosfera_2016 108

ANTEZANA, F. L. Crescimento inicial de 15 espécies nativas do bioma cerrado sob diferentes condições de adubação e roçagem, em Planaltina - DF. 2008. 84f. Dissertação (Mestrado em Ciências Florestais) Universidade de Brasília, Brasília, 2008.

ARAÚJO, L. H. B. de; NÓBREGA, C. C., da; SILVA, R. A. R.; GUERRA, C. A. G.; SANTANA, J. A. da S. Comportamento de mudas de espécies florestais em projeto de Restauração Ecológica sobre área de dunas em Natal, RN. ACSA- Agropecuária Científica no Semiárido, Campina Grande, v. 10, n. 1, p. 122-127, 2014. DOI: http://dx.doi.org/10.30969/acsa.v10i1.450

BARBOSA, P. T. F.; BARBOSA, P. T. F.; MORAIS, M. A. de; SOUZA, M. M. de. Recuperação de mata ciliar na Fazenda Pinhal em Passos / MG. Revista Agrogeoambiental, Pouso Alegre, n. 1, p. 73-77, 2013. DOI: http://dx.doi.org/10.18406/2316-1817v1n12013583

BATISTA, J. L. F.; BATISTA, J. L. F.; COUTO, H. T. Z. do; FILHO, D. F. da S. Monitoramento arborimétrico. In: Quantificação de recursos florestais. 1. ed. São Paulo: Oficina de Textos, 2014. p. 103-124.

BILIA, D. A. C.; BARBEDO, C. J.; CICERO, S. M.; MARCOS FILHO, J. Ingá: uma espécie importante para recomposição vegetal em florestas ripárias, com sementes interessantes para a ciência. Informativo ABRATES, Londrina, v. 13, p. 26-30, 2003.

BONNET, A.; CURCIO, G. R. Uso de espécies arbóreas nativas para a propriedade rural e mercado regional na região de Goiânia, GO. Colombo: Embrapa, 2016. 15 p. (Comunicado Técnico, 379)

BRANCALION, Pedro HS; GANDOLFI, Sergius; RODRIGUES, Ricardo Ribeiro. Restauração florestal. 1. ed. São Paulo: Oficina de textos, 2015. 432 p.

BRASIL. Lei $\mathrm{n}^{\circ} 12.651$, de 25 de maio de 2012. Diário oficial da União, Brasília, 2012.

BRASIL Ministério do Meio Ambiente. Cerrado brasileiro tem $\overline{\mathbf{5 4}, 5 \%}$ vegetação preservada. 2015 . Disponível em: $<$ http://www.brasil.gov.br/meioambiente/2015/11/cerrado-brasileiro-tem-54-5-davegetacao-preservada $>$. Acesso em: 21 maio 2018.

CARDOSO, M. R. D.; MARCUZZO, F. F. N.; BARROS, J. R. Classificação climática de köppen-geiger para o Estado de Goiás e o Distrito Federal. Acta Geográfica, Boa Vista, v. 8, n. 16, p. 40-55, 2014.

CARNEVALI, N. H. DE S.; Sobrevivência e crescimento inicial de espécies arbóreas nativas implantadas em pastagem degradada. Floresta, Curitiba, v. 46, n. 2, p. 277-286, 2016. http://dx.doi.org/10.5380/rf.v46i2.42881

CARVALHO, P. E. R. Espécies Arbóreas Brasileiras. v. 1 ed. Colombo/PR: Embrapa Florestas, 2003.

CARVALHO, P. E. R. Espécies Arbóreas Brasileiras. v. 2 ed. Colombo/PR: Embrapa Florestas, 2006.

CARVALHO, P. E. R. Espécies Arbóreas Brasileiras. v. 3 ed. Colombo/PR: Embrapa Florestas, 2008.

CARVALHO, P. E. R. Farinha-Seca Albizia niopoides. Colombo: Embrapa Florestas. 8 p. (Comunicado Técnico, 226)

CLAASSEN, V. P.; CAREY, J. L. Comparison of slowrelease nitrogen yield from organic soil amendments and chemical fertilizers and implications for regeneration of disturbed sites. Land Degradation \& Development, Chichester, v. 18, n. 2, p. 119-132, mar. 2007. DOI: https://dx.doi.org/10.1002/ldr.770

CORTES, J. M. Desenvolvimento de espécies nativas do cerrado a partir do plantio de mudas e da regeneração natural em uma área em processo de recuperação, Planaltina-DF. 2012. 89f. Dissertação (Mestrado em Ciências Florestais) - Universidade de Brasília, Brasília, 2012.

DARONCO, C.; GALVÃO DE MELO, A. C.; DURIGAN, G. Ecossistema em restauração versus ecossistema de referência: estudo de caso da comunidade vegetal de mata ciliar em região de Cerrado, Assis, SP, Brasil. Hoehnea, São Paulo, v. 40, n. 3, p. 485-498, 2013. DOI: http://dx.doi.org/10.1590/S2236-89062013000300008

DUBOC, E.; GUERRINI, I. A. Crescimento inicial e sobrevivência de espécies florestais de matas de galeria no domínio do cerrado em resposta a fertilização. Energia na Agricultura, Botucatu, v. 22, n. 1, p. 42-60, 2007

DURIGAN, G. Restauração da Cobertura Vegetal em Região de Domínio do Cerrado. In: GALVÃO, A.P.M.; PORFÍRIO-DA-SILVA, V. (Org.). Restauração Florestal: Fundamentos e Estudos de Caso. Colombo: Embrapa Florestas, 2005. p. 103-118.

DURIGAN, G.; MELO, A. C. G. DE; MAX, J. C. M. Manual para recuperação da vegetação de cerrado. 3 . ed. São Paulo: SMA/SP, 2011. 26 p.

FAO_Food and Agriculture Organization of the United Nations; ITPS_Intergovernmental Technical Panel on Soils. Status of the World's Soil Resources (SWSR) Main Report. Rome, Italy: FAO/ITPS, 2015.

FELFILI, J. M. ; RIBEIRO, J. F.; FAGG, C. W.; MACHADO, J. W. B. Recuperação De Matas De Galeria. Planaltina: Embrapa Cerrados, 2000. 45 p.

FERREIRA, W. C.; BOTELLHO, S. A.; DAVIDE, A. C.; FARIA, J. M R. Estabelecimento de mata ciliar às margens do reservatório da usina hidrelétrica de Camargos, MG. Ciência Florestal, v. 19, n. 1, p. 69-81, 2009. DOI: http://dx.doi.org/10.5902/19805098421

GUSSON, A. E. et al. Interferência do aumento de umidade do solo nas populaçõ es de Myracrodruon urundeuva Allemão e Anadenanthera colubrina (Vell.) Brenan em reservatórios artificiais de Usinas Hidrelétricas. Scientia Forestalis, Piracicaba, v. 39, n. 89, p. 35-41, 2011.

LIMA, J. A. DE; SANTANA, D. G. DE; NAPPO, M. E. Comportamento inicial de espécies na revegetação da mata de galeria na Fazenda Mandaguari, em Indianópolis, MG. Revista Árvore, Viçosa, v. 33, n. 4, p. 685-694, ago. 2009. DOI: http://dx.doi.org/10.1590/S010067622009000400011

LORENZI, H. Árvores Brasileiras. v. 1 ed. Nova Odessa, SP: Editora Plantarum, 1992. 352 p.

LORENZI, H. Árvores Brasileiras. 2 ed. v.2 ed. Nova Odessa, SP: Editora Plantarum, 1998.

MARTINOTTO, F.; MARTINOTTO, C.; COELHO, M. de F. B.; AZEVENDO, R. A. B. de; ALBUQUERQUE, M. C. de F e. Sobrevivência e crescimento inicial de espécies arbóreas nativas do Cerrado em consórcio com mandioca. Pesquisa Agropecuária Brasileira, Brasília, v. 47, n. 1, p. 22-29, jan. 2012.

MARTINS, K. G.; MARQUES, M. C. M.; SANTOS, E. dos; MARQUES, R. Effects of soil conditions on the diversity 
of tropical forests across a successional gradient. Forest Ecologyand Management, Amsterdam, v. 349, p. 4-11, 2015 .

DOI:

https://dx.doi.org/10.1016/j.foreco.2015.04.018

MEIRA JUNIOR, M. S. de; PEREIRA, I. M.; MACHADO, E. L. M.; MOTA, S. da L. L.; OTONI, T. J. O. Espécies potenciais para recuperação de áreas de Floresta Estacional Semidecidual com exploração de minério de ferro na Serra do Espinhaço. Bioscience Journal, Uberlândia, v. 31, n. 1, p. 283-295, 2015. DOI: http://dx.doi.org/10.14393/BJ-v31n1a2015-23414

MEWS, H. A. O Cerrado sentido restrito sobre dois substratos no Brasil Central: padrões da flora lenhosa e correlações com variáveis geoedafoclimáticas. 2014. 78f. Tese (Doutorado em Ciências Florestais) Universidade de Brasília, Brasília, 2014.

MYERS, N. ; MITTERMEIER, R. A. ; MITTERMEIER, C. G. ; FONSECA, G. A. B. da ; KENT, J. Biodiversity hotspots for conservation priorities. Nature, London, v. 403 , n. 6772, p. 853-858, fev. 2000. DOI: https://dx.doi.org/10.1038/35002501

NERI, A. V. ; SOARES, M. P. ; MEIRA NETO, J. A. A. ; DIAS, L. E. Espécies de cerrado com potencial para recuperação de áreas degradadas por mineração de ouro, Paracatu-MG. Revista Árvore, Viçosa, v. 35, n. 4, p. 907-918, ago. 2011. DOI: http://dx.doi.org/10.1590/S0100-67622011000500016

NEWBOLD, T; HUDSON, L. N.; ARNELL, A. P.; CONTU, S.; PALMA, A. de; FERRIER, S.; HILL, S. L. L.; HOSKINS, A. J.; LYSENCO, I.; PHILLIPS, H. R. P.; BURTON, V. J.; CHNG, C. W. T.; EMERSON, S.; GAO, D.; PASK-HALE, G.; HUTTON, J.; JUNG, M.; SANCHEZ-ORTIZ, K.; SIMMONS, B. I.; WHITMEE, S.; ZHANG, H.; SCHARLEMANN, J. P. W.; PUVIS, A. Has land use pushed terrestrial biodiversity beyond the planetary boundary? A global assessment. Science, v. 353 , n. 6296, p. 288-291, jul. 2016. DOI: https://dx.doi.org/10.1126/science.aaf2201

OLIVEIRA, M. C.; PASSOS, F. B.; RIBEIRO, J. F.; AQUINO, F. G.; OLIVEIRA, F. F.; SOUSA, S. R. Crescimento de espécies nativas em um plantio de recuperação de Cerrado sentido restrito no Distrito Federal, Brasil. Revista Brasileira de Biociências, Porto Alegre, v. 13, n. 1, p. 25-32, 2015.

PEREIRA, I. M.; BOTELHO, S. A.; DAVIDE, A. C. Restauração de ecossistemas: Bases ecológicas e silviculturais. In: DAVIDE, A. C.; BOTELHO, S. A. (Ed.). Fundamentos e Métodos de Restauração de Ecossistemas Florestais - 25 anos de experiência em Matas Ciliares. Lavras: UFLA, 2015. p. 369-432.

PIÑA-RODRIGUES, F. C. M. LOPES, L.; BLOOMFIELD, V. K. Análise do desenvolvimento de espécies arbóreas da Mata Atlântica em sistema de plantio adensado para a revegetação de áreas degradadas em encosta, no entorno do Parque Estadual do Desengano Ouro Preto, MG. In: SIMPÓSIO NACIONAL DE RECUPERAÇÃO DE ÁREAS DEGRADADAS, 3., 1997, Ouro Preto. Anais... Ouro Preto: SINRAD, 1997. p. 283-291.

PIRES, H. R. DE A. Efeito do alagamento na germinação de sementes e no crescimento de plantas jovens de Genipa americana e Guazumaulmifolia com ocorrência no Cerrado e na Amazônia Efeito do alagamento na germinação de sementes e no crescimento de plantas jovens de Genipa ulmifolia com ocorrência no Cerrado e na Amazônia. 2015. 57f. Dissertação (Mestrado em Botânica) - Universidade de Brasília, Brasília, 2015.

POTT, A.; POTT, V. J. Plantas nativas para recuperação de áreas degradas e reposição de vegetação em Mato Grosso do Sul. Campo Grande: Embrapa Gado de Corte, 2002. 6 p. (Comunicado Técnico, 75).

PUIG, H. Biologia e morfologia. In: PUIG, H. A Floresta Tropical Úmida. São Paulo: UNESP, 2008. p. 119-252.

REATTO, A. et al. Solos de ocorrência em duas áreas sob Matas de Galeria no Distrito Federal: aspectos pedológicos, uma abordagem química e físico-hídrica. In: RIBEIRO, J. F.; FONSECA, C. E. L. DA; SOUSASILVA, J. C. (Ed.). Cerrado: caracterização e recuperação de Matas de Galeria. Planaltina: Embrapa Cerrados, 2001. p. 115-140.

REZENDE, R. P. Recuperação de Matas de Galeria em propriedades rurais do Distrito Federal e Entorno. 2004. 145f. Dissertação (Mestrado em Ciências Florestais) - Universidade de Brasília, Brasília, 2004.

SANTOS, D. L. DOS; SUGAHARA, V. Y.; TAKAKI, M. Efeitos da luz e da temperatura na germinação de sementes de Tabebuia serratifolia (Vahl) Nich, Tabebuiachrysotricha (Mart. ex DC.) Standl. E Tabebuiaroseo-alba (Ridl) Sand - Bignoniaceae. Ciência Florestal, Santa Maria, v. 15, n. 1, p. 87-92, 2005. DOI: http://dx.doi.org/10.5902/198050981826

SILVA, L. de C. R.; CORRÊA, R. S. Sobrevivência e crescimento de seis espécies arbóreas submetidas a quatro tratamentos em área minerada no cerrado. Revista Árvore, Viçosa, v. 32, n. 4, p. 731-740, ago. 2008. DOI: http://dx.doi.org/10.1590/S0100-67622008000400015

SILVA JÚNIOR, M. C.; FELFILI, J. M.; NOGUEIRA, P. E.; REZENDE, A. V. Análise florística das matas de galeria no Distrito Federal. In: RIBEIRO, J. F. Cerrado: matas de galeria. Planaltina: Embrapa/CPAC, 1998. p. 52-84.

SILVA JÚNIOR, M. C.; FELFILI, J. M.; WALTER, B. M. T.; NOGUEIRA, P. E.; REZENDE, A. V.; MORAIS, R. D. O.; NÓREGA, M. G. G. Análise da flora arbórea de Matas de Galeria no Distrito Federal: 21 levantamentos. SOUSA-SILVA, J. C. Cerrado: caracterização e recuperação de matas de galeria. Planaltina, Embrapa Cerrados, p. 143-191, 2001.

SOLLINS, P. Factors Influencing Species Composition in Tropical Lowland Rain Forest: Does Soil Matter? Ecology, New York, v. 79, n. 1, p. 23-30, jan. 1998. DOI: https://dx.doi.org/10.1890/00129658(1998)079[0023:FISCIT]2.0.CO;2

SWANSON, F. J. KRATZ, T. K.; CAINE, N.; WOODMANSEE, R. G. Landform Effects on Ecosystem Patterns and Processes. BioScience, v. 38, n. 2, p. 92-98, fev. 1988. DOI: https://dx.doi.org/10.2307/1310614

VITOUSEK, P. M.; PORDER, S.; HOULTON, B. Z.; CHADWICK, O. A. Terrestrial phosphorus limitation: mechanisms, implications, and nitrogen-phosphorus interactions. Ecological Applications, v. 20, n. 1, p. 515, jan. 2010. DOI: https://dx.doi.org/10.1890/08-0127.1

WALTER, B. M. T.; CARVALHO, A. M. DE; RIBEIRO, J. F. O conceito de savana e de seu componente Cerrado. In: SANO, S. M.; ALMEIDA, S. P. DE; RIBEIRO, J. F. (Ed.). Cerrado: ecologia e flora. Brasília: Embrapa Informação Tecnológica, 2008. p. 21-45. 